\title{
Autoacetylation of Purified Calreticulin Transacetylase Utilizing Acetoxycoumarin as the Acetyl Group Donor
}

\author{
Seema Bansal • Prija Ponnan • Hanumantharao G. Raj • \\ Susan T. Weintraub • Madhu Chopra • Ranju Kumari • \\ Daman Saluja • Ajit Kumar • Tapesh K. Tyagi • \\ Prabhjot Singh • Ashok K. Prasad • Luciano Saso • \\ Ramesh C. Rastogi • Virinder S. Parmar
}

Published online: 18 November 2008

(C) Humana Press 2008

\section{Erratum to: Appl Biochem Biotechnol \\ DOI 10.1007/s12010-008-8357-2}

In the original paper, the figures were incorrectly labeled. The figures and their corresponding legends should appear as follows.

Figures 1, 2, 3, 4, 5 and 6

\footnotetext{
The online version of the original article can be found at http://dx.doi.org/10.1007/s12010-008-8357-2

$\overline{\text { S. Bansal } \cdot \text { P. Ponnan } \cdot \text { H. G. Raj }}(\bowtie) \cdot$ R. Kumari $\cdot$ A. Kumar $\cdot$ T. K. Tyagi $\cdot$ P. Singh Department of Biochemistry, V. P. Chest Institute, University of Delhi, Delhi 1100 07, India e-mail: rajhg@yahoo.com

P. Ponnan · A. K. Prasad • R. C. Rastogi • V. S. Parmar

Department of Chemistry, University of Delhi, Delhi 1100 07, India

M. Chopra · D. Saluja

Dr.B.R.Ambedkar Center for Biomedical Research, University of Delhi, Delhi 110007 Delhi, India

S. T. Weintraub

Department of Biochemistry, The University of Texas Health Science Center, 7703 Floyd Curl Drive, San Antonio, TX 78229-3900, USA

L. Saso

Department of Human Physiology and Pharmacology,

"Vittorio Erspamer" Sapienza University of Rome, P.le Aldo Moro 5, 00185 Rome, Italy

茫 Humana Press
} 


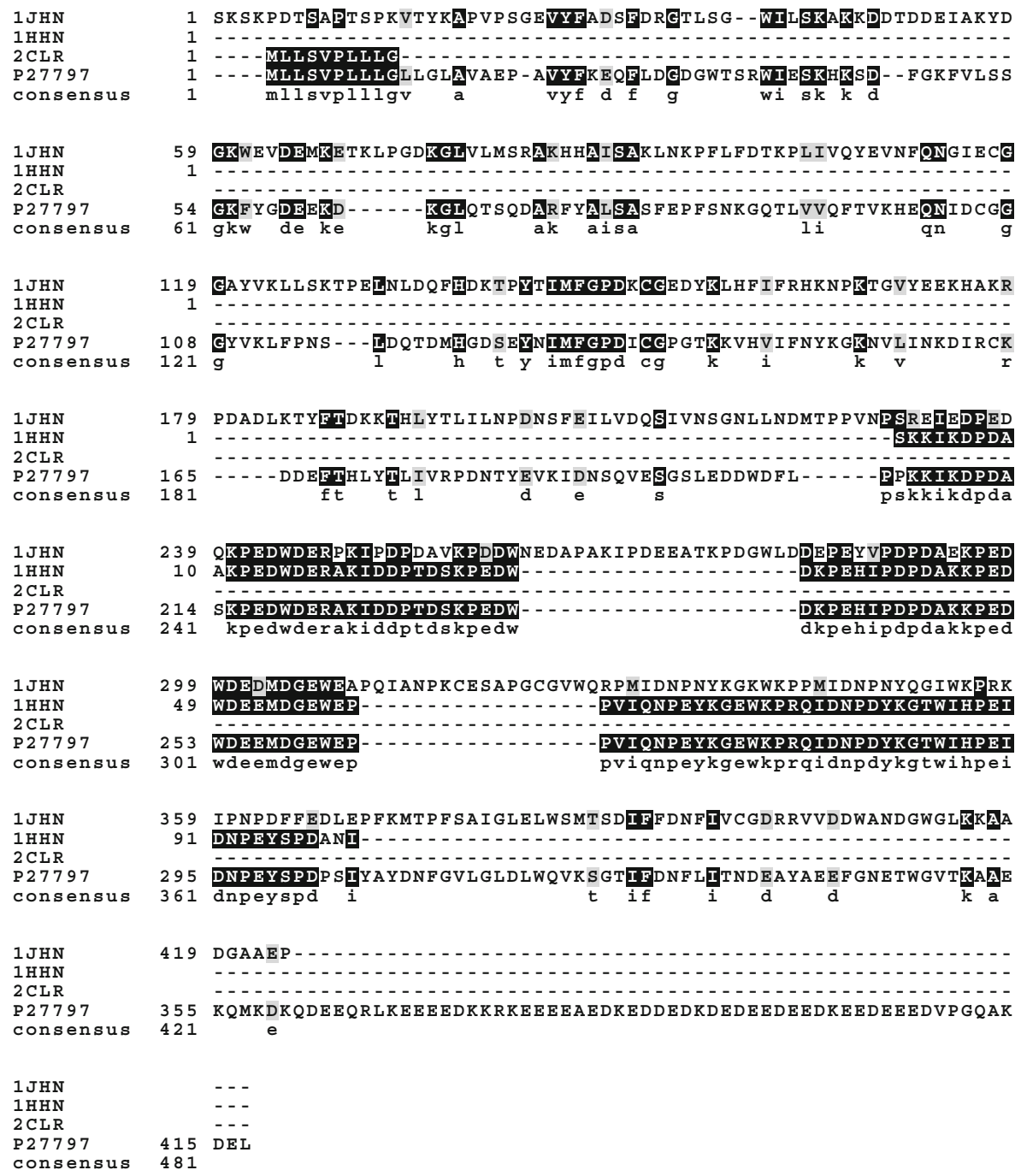

Fig. 1 Sequences of calnexin (PDB ID 1JHN), CR P-domain (PDB ID 1HHN), CR signal peptide sequence (PDB ID 2CLR) and human CR sequence swissprot accession no. P27797 are aligned using InsightII/ Homology and displayed by BOXSHADE program (http://www.ch.embnet.org/software/BOX_form.html). Shaded residues denote identity (Black) or close similarity (Gray) between sequences 


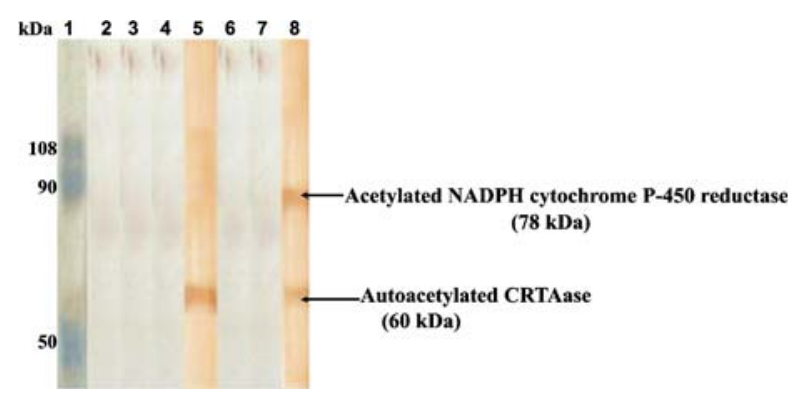

Fig. 2 Effect of $\mathrm{Ca}^{2+}$ on CRTAase catalyzed acetylation of NADPH cytochrome P-450 reductase by DAMC. Lane 1 Prestained molecular weight markers; lane 2 CRTAase +DMSO; lane 3 NADPH cytochrome $\mathrm{P}-450$ reductase + DAMC; lane 4 CRTAase $+\mathrm{DMSO}+\mathrm{CaCl}_{2}(5 \mu \mathrm{M})+\mathrm{NADPH}$ cytochrome P-450 reductase; lane 5 CRTAase + DAMC; lane 6 CRTAase $+\mathrm{CaCl}_{2}(5 \mu \mathrm{M})+$ DAMC; lane 7 CRTAase $+\mathrm{CaCl}_{2}(5 \mu \mathrm{M})+$ DAMC + NADPH cytochrome P-450 reductase; lane 8 CRTAase + DAMC + NADPH cytochrome P-450 reductase 

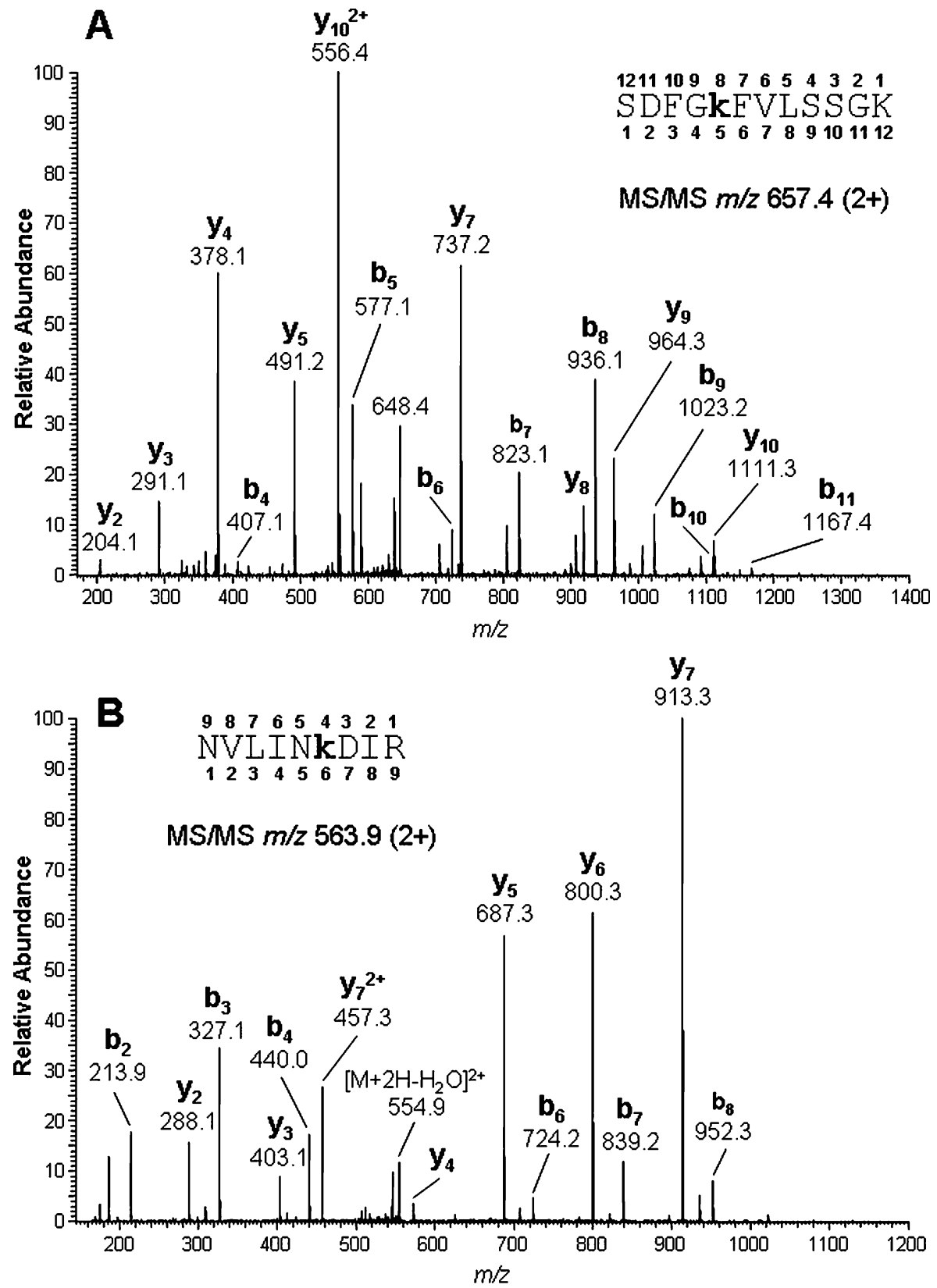

Fig. 3 Tandem mass spectra of selected CR tryptic peptides containing acetyl-lysine. residue numbers shown in boldface type above and below the peptide sequences indicate detected collision-induced dissociation fragments for y-series (C-terminal) and b-series (N-terminal) ions, respectively. (a) Sequence SDFGkFVLSSGK (Corresponding to residues 45-55 of CR) observed in the tryptic digest of CR, the ion at $\mathrm{m} / \mathrm{z} 648.4$ was generated by loss of water from doubly charged precursor; (b) sequence NVLINkDIR. (Corresponding to residues 154-162 of CR) peak at m/z 554.9 represents the ion due to loss of water in the doubly charged precursor; (c) sequence kIKDPDASKPEDWDER (Corresponding to residues 207-222 of $\mathrm{CR}$ ) observed in the tryptic digest of CR. MS/MS m/z 657.9 (3+). Asterisk corresponds to internal fragments 


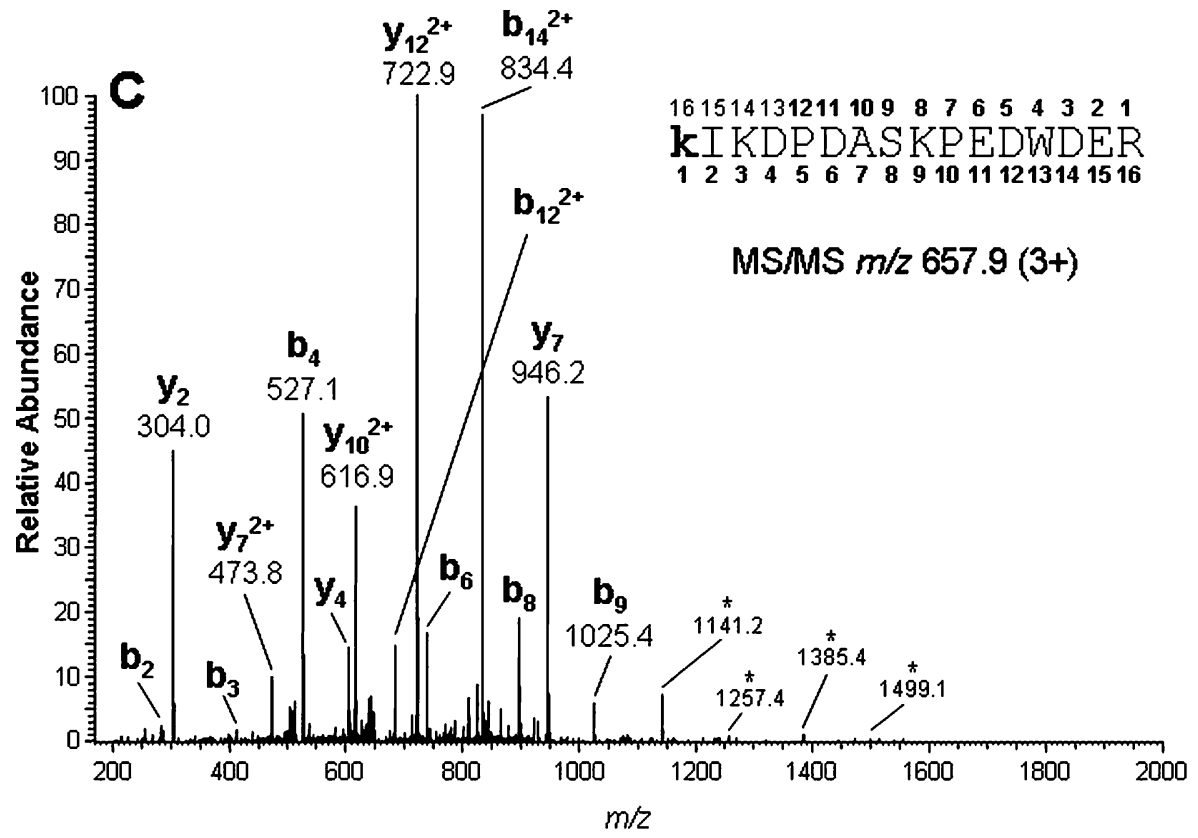

Fig. 3 (continued)

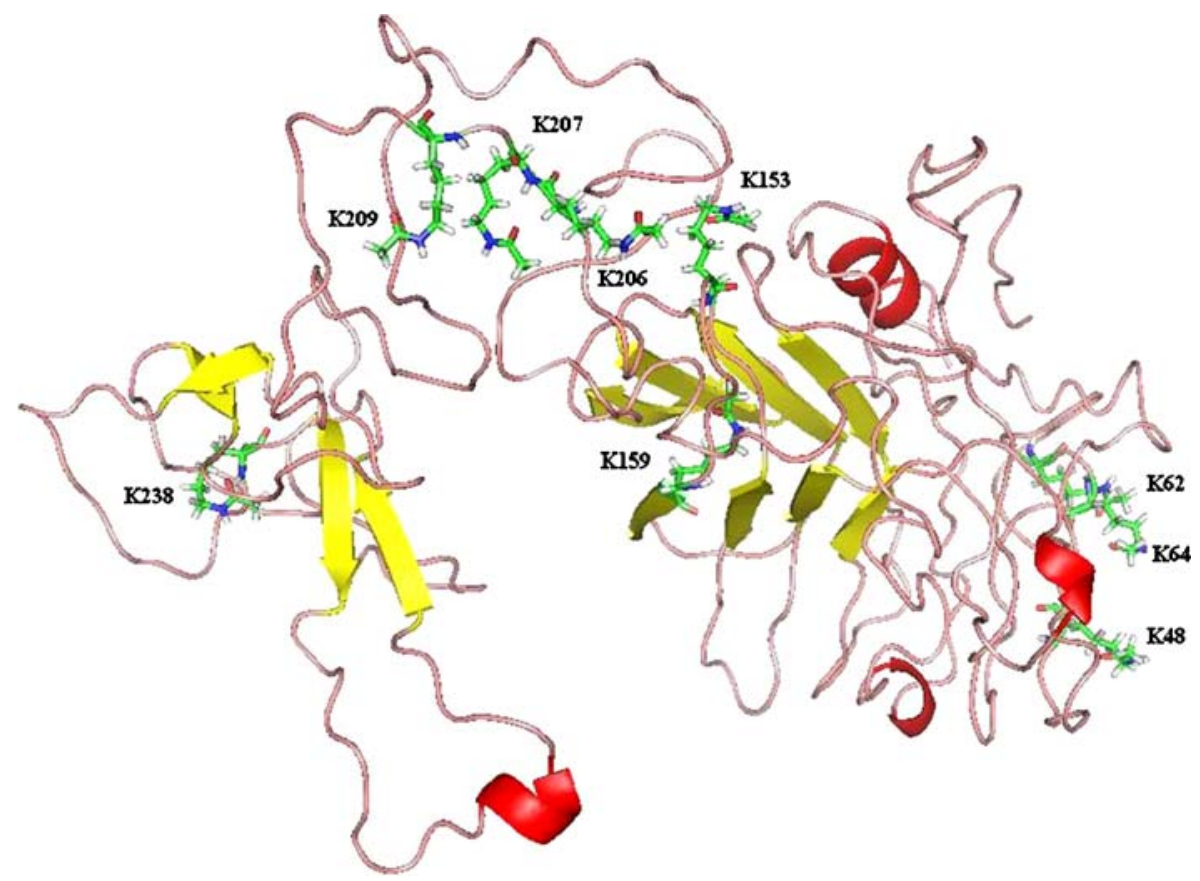

Fig. 4 The predicted structure of CR is represented in Kabsch-Sander secondary structure with $\alpha$-helices in red and $\beta$-sheets in yellow. The modified lysine residues are in stick representation with standard atom colors. The figure was prepared using Pymol software [43] 


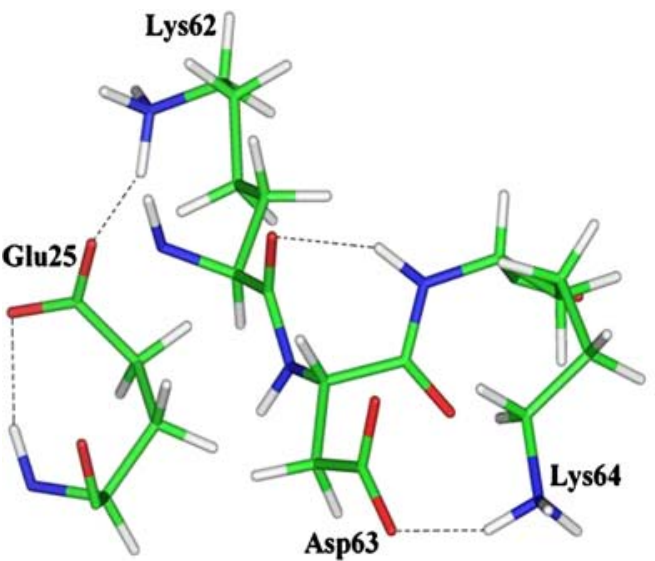

A

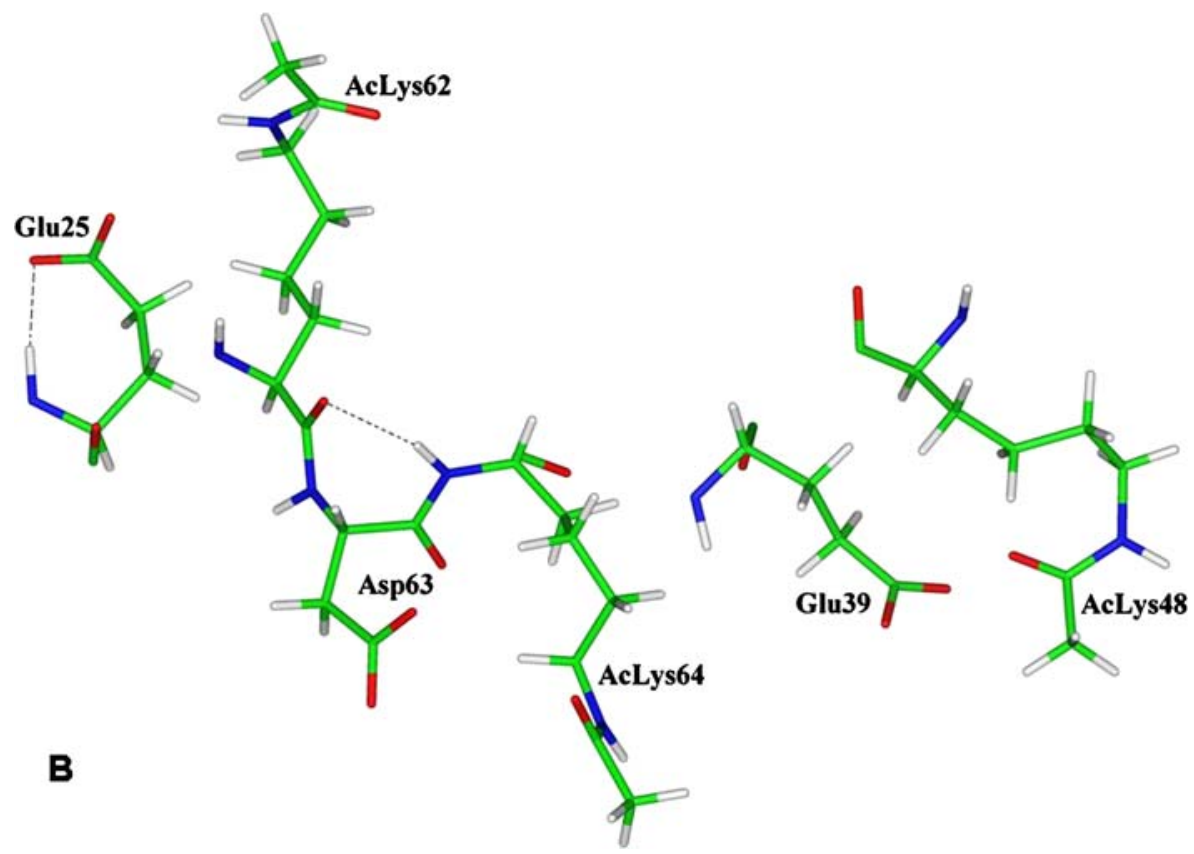

Fig. 5 Comparison of intermolecular H-bonds (dotted lines) of lysines with glutamate and aspartate residues in non-acetylated (a) and acetylated (b) CR, after energy minimization. The amino acid residues are rendered in sticks with standard atom colors. Loss of H-bonds between Lys-62 and Glu-25, Lys-64 and Asp-63 also in Lys-48 and Glu-39 was observed due to neutralization of the positively charged lysine residue by acetylation. The acetylated lysine residues of N-domain are highlighted here. The figure was generated using InsightII program [26]

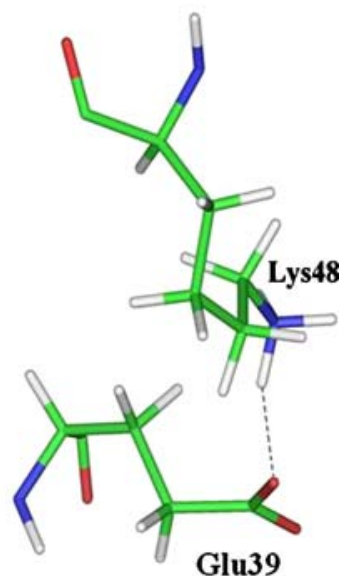


A
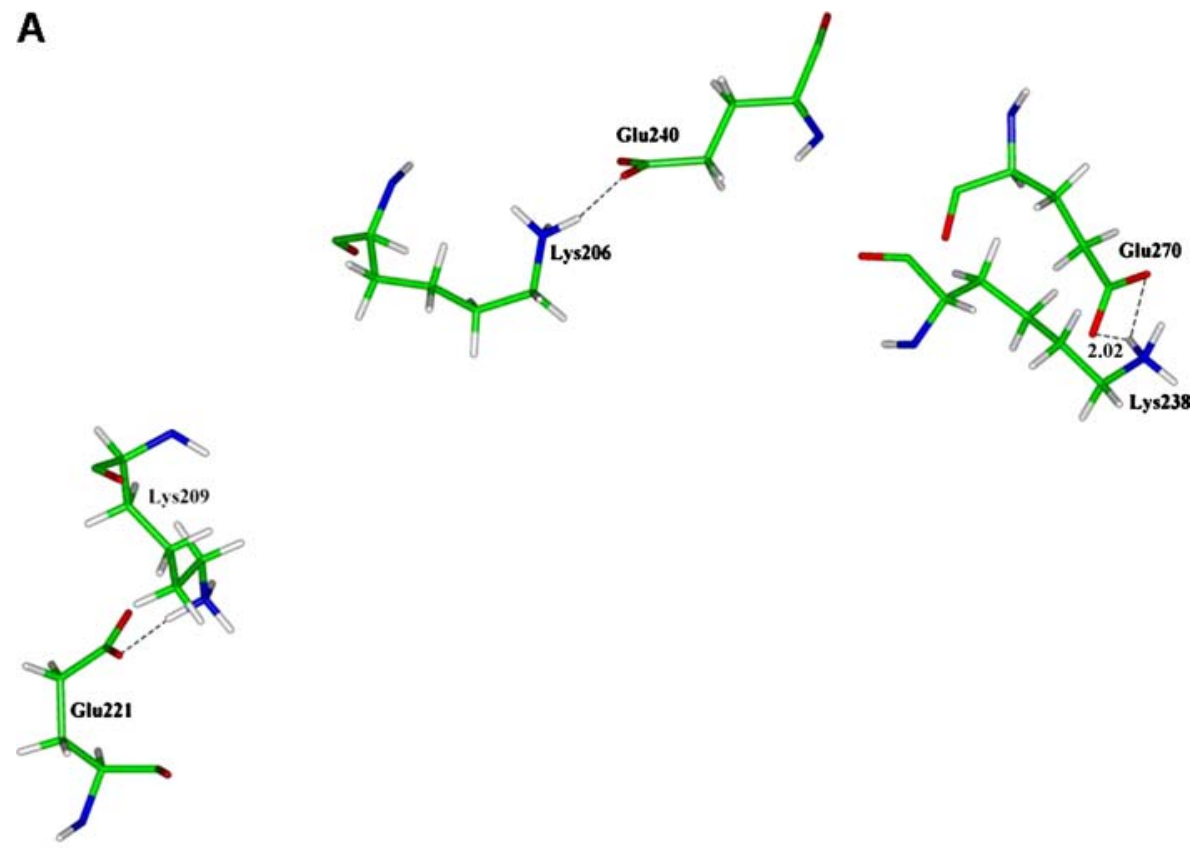

B
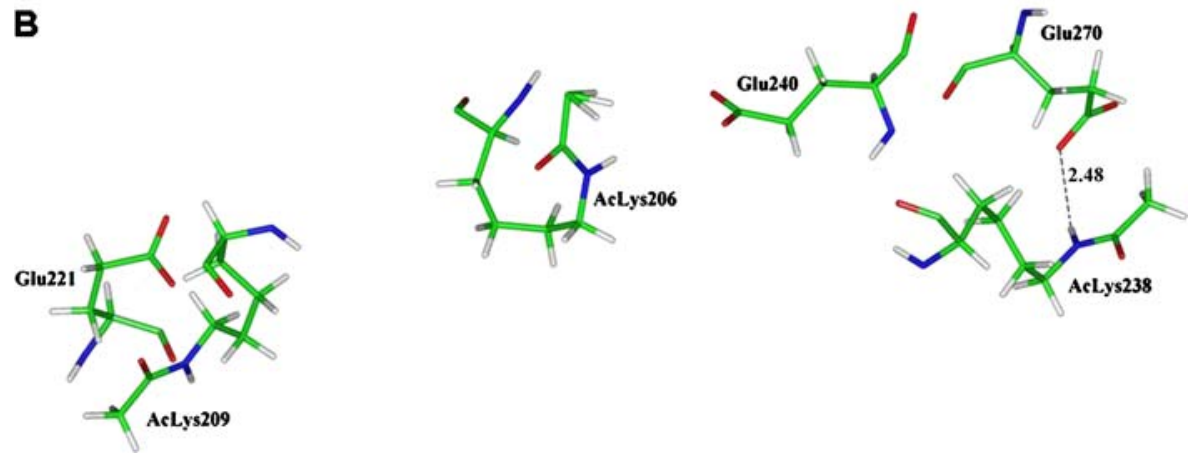

Fig. 6 Comparison of non-acetylated (a) and acetylated (b) CR P-domain lysines. Loss of H-bonds between Lys-206 and Glu-240, Lys-238 and Glu-270 as well as Lys-209 and Glu-221 was observed due to $N-\varepsilon-$ acetylation of lysine residues. Also, increase in the intermolecular distances between acetyl-lysine (AcLys238) and glutamate (Glu-270) is observed. The amino acid residues are rendered in sticks with standard atom colors. The amino acid residues rendered in sticks with standard atom colors and figure is realized using InsightII program [26] 